\title{
Network Analysis of Herbs Recommended for the Treatment of COVID-19
}

\author{
Lin Ang $\mathbb{D}^{1,2}$ \\ Hye Won Lee $\mathbb{D}^{3}$ \\ Anna Kim (iD ${ }^{4}$ \\ Jun-Yong Choi $\mathbb{D}^{5}$ \\ Myeong Soo Lee $\mathbb{I}^{1,2}$ \\ 'Clinical Medicine Division, Korea \\ Institute of Oriental Medicine, Daejeon, \\ Korea; ${ }^{2}$ Korean Convergence Medicine, \\ University of Science and Technology, \\ Daejeon, Korea; ${ }^{3} \mathrm{Herbal}$ Medicine \\ Research Division, Korea Institute of \\ Oriental Medicine, Daejeon, Korea; \\ ${ }^{4}$ Future Medicine Division, Korea \\ Institute of Oriental Medicine, Daejeon, \\ Korea; ${ }^{5}$ Pulmonology Division, School of \\ Korean Medicine and Korean Medicine \\ Hospital of Pusan National University, \\ Yangsan, Korea
}

Purpose: In this study, we aimed to identify the pattern and combination of herbs used in the formulae recommended for treating different stages of COVID-19 using a network analysis approach.

Methods: The herbal formulae recommended by official guidelines for the treatment of COVID-19 are included in the present analysis. To describe the tendency of herbs to form a "herb pair", we computed the mutual information (MI) value and distance-based mutual information model (DMIM) score. We also performed modularity, degree, betweenness, and closeness centrality analysis. Network analyses were performed and visualized for each disease stage.

Results: A total of 142 herbal formulae comprising 416 herbs were analyzed. All possible herbal pairs were examined, and the top frequently used herbal pairs were identified for each disease stage. The herb Glycyrrhizae radix et rhizoma is only identified in one herb pair, even though this herb is identified as one of the herbs with high frequency of use for every disease stage. This suggests that the DMIM score could be used to identify the optimal combination rule of herbal formulae by achieving a balance among the herbs' frequency and relative distance in herbal formulae.

Conclusion: Our results presented the prescription patterns and herbal combinations of the herbal formulae recommended for the treatment of COVID-19. This study may provide new insights and ideas for clinical research in the future.

Keywords: coronavirus 19, disease stage, herbs pairing, prescription pattern, network graph

\section{Introduction}

The coronavirus disease responsible for the current pandemic is an acute respiratory illness caused by a new strain of coronavirus (COVID-19). As of 29 March 2021, COVID-19 has affected approximately 127 million people worldwide and the number is still growing every day. ${ }^{1}$ COVID-19 is classified into four stages based on their clinical manifestation: the mild stage is classified by mild symptoms such as fever and respiratory symptoms with no signs of radiographic findings of pneumonia; the moderate stage by similar symptoms of fever and respiratory symptoms with radiographic findings of pneumonia; the severe stage by the onset of respiratory failure requiring mechanical ventilation; and the recovery stage.

Although both Food and Drug Administration (FDA)-approved antiviral drugs and several types of vaccines are currently available, many alternative treatments are still being proposed as an adjunctive treatment for COVID-19. It has been reported that herbal medicines could be considered an alternative approach for the treatment and prevention of COVID-19., ${ }^{2,3}$ Recent systematic review articles also 
concluded that herbal medicine showed significant effects in increasing the total effective rate and alleviating the symptoms. ${ }^{4-6}$

Network science is a field where complex networks are studied and represented by nodes and edges. ${ }^{7}$ Network analysis, derived from network science, uses the mathematical language of graph theory to depict relationships among analyzed variables, and such analyses are often presented using visualization techniques. ${ }^{8}$ In recent years, network analysis has been used in the field of complementary and alternative medicine. Several acupuncture studies used complex network analysis to examine the distribution pattern of acupoints selection and the regularities of acupoint combinations. ${ }^{9,10}$ Herbal studies also integrate complex network analysis techniques to investigate herb-target interactions and herbal combination regularities among herbal formulae. ${ }^{11-14}$

In this study, we applied network analysis to determine the prescription pattern and combination of herbs used in the herbal formulae recommended for the treatment of COVID19. To analyze the distribution pattern of herbs, we computed the data extracted from available treatment guidelines and visualized the network based on each disease stage.

\section{Materials and Methods}

\section{Data Sources}

In our previous study, ${ }^{15}$ we collected and summarized the composition of herbs used in the herbal formulae recommended by the official guidelines for the treatment of COVID-19 (26 Chinese guidelines and two Korean guidelines), which comprised a total of 142 herbal formulae with 416 herbs. The names of the herbs are standardized based Pharmacopoeia of the People's Republic of China (Appendix 1). To identify useful herbal combinations used in the treatment of COVID-19 for each disease stage, we included all the herbal formulae and herbs of our previous study in this network analysis.

\section{Network Analysis}

Network analyses were performed using Netminer v.4.4.3. b (Cyram Inc, Seoul, Korea) for each disease stage.

\section{Mutual Information (MI) Value and Distance-Based Mutual Information Model (DMIM) Score}

The mutual information (MI) value between herbs $x$ and $y$ was calculated using $M I(x, y)=\mathrm{P}(x, y) * \log \left(\frac{\mathrm{P}(\mathrm{x}, \mathrm{y})}{\mathrm{P}(\mathrm{x}) * \mathrm{P}(\mathrm{y})}\right)$.
$\mathrm{P}(x, y)=\left(\sum_{i=1}^{m} I(x, y, i)\right) / m$ is the coincidence frequency of $x$ and $y$, where $m$ is the total number of formulas and $I(x$, $y, i)$ is the indicator function of $x$ and $y$ that shows whether herb $x$ and $y$ were used together in formula $i$. Here, $(x)=$ $\sum_{i=1}^{m} I(x, i) / m$ is the frequency of herb $x$ and this formula also applies to $\mathrm{P}(y)$. The higher the value of MI between the herbs, the stronger the correlation between them is. ${ }^{16}$

Distance-based mutual information model (DMIM) scores between herbs $x$ and $y$ were defined as score $(x, y)=M I(x, y) / d(x, y)$. DMIM combines the MI value and the average distance between herbs, where the distance between herb $x$ and herb $y$ in the $i$ th formula was defined as $d(x, y, i)=|B(x, i)-B(y, i)|$. The average distance of herb $x$ and herb $y$ in the dataset was computed using $\quad d(x, y)=\sum_{i=1}^{m}(I(x, y, i) * d(x, y, i)) / \sum_{i=1}^{m}(x, y, i)$. DMIM describes the tendency of herb $x$ and herb $y$ to form a herb pair. ${ }^{16}$ Similarly, the higher the DMIM score between the herbs, the higher the tendency of the herbs to form a pair.

\section{Modularity Analysis}

To analyze the modular structure of the herb combination network, the algorithm introduced by Newman, ${ }^{17}$ which maximizes modularity with a greedy approach, was applied to a high-frequency herb pair matrix. The modularity $(Q)$ can be written as $Q=(1 / 4 m) \sum i j[A i j-P i j](g i, g j)$, where $A i j=1$ if there is an edge joining node $i$ and $j$ and 0 ; otherwise, $\mathrm{P} i j$ was defined as $k_{i} k_{j} / 2 \mathrm{~m} . k_{i}$ and $K_{j}$ are the degree of each node and $m$ is the number of edges in the networks; $\delta(\mathrm{g} i, \mathrm{~g} j)=1$ if node $i$ and $j$ belong to the same group and $\delta(\mathrm{g} i, \mathrm{~g} j)=0$ otherwise. Modules are then formed for the nodes that are highly interconnected among themselves.

\section{Degree, Betweenness, and Closeness Centrality Analysis}

We computed the degree, betweenness, and closeness centralities for all nodes (herbs) at each disease stage. The degree of centralization index defines the variability of individual centrality scores. The higher the degree of centralization index is, the more centralized the overall network is. The betweenness centrality defines the extent to which a node lies between all other pairs of nodes on their 
paths. Here, the more times a node appears in the paths, the higher its centrality is. The closeness centralization index is a measure of the variability of individual closeness centrality scores. The higher the closeness index, the shorter the average distance of the node to all other nodes. ${ }^{18}$

\section{Network Visualization}

In this study, Netminer v4.4.3.b. (Cyram Inc, Seoul, Korea) was used to generate the networks to visualize relationships among herbs. We used a spring layout that uses dynamic theory to visualize the degree and betweenness centrality analysis for different stages of COVID-19. Each module is presented in a different color for clarity purposes. The size of the nodes is depending on the number of frequency and the density of the edges between the nodes represent the strength of their correlation.

\section{Results}

This study used DMIM techniques to examine the relationships among the 416 herbs included. All possible herbal pairs were examined and the most frequently used herbal pairs were identified for each disease stage. We also identified the patterns in the combinations of herbs and illustrated the modular structure using a spring layout.

\section{Mild Stage}

Of the 36 herbal formulae with a total of 90 herbs analyzed in this stage, we identified 12 herb pairs with the highest frequency of use (DMIM score $>0.69$ ). The six herb pairs with the highest DMIM score were Atractylodis rhizoma-Amomi tsao-ko fructus, Arecae semen-Amomi tsao-ko fructus, Pinelliae rhizoma-Amomi fructus rotundus, Coicis semen-Amomi fructus rotundus, Massa medicata fermentata-Crataegi fructus, and PolyporusAlismatis rhizoma (Table 1).

The frequency, degree, betweenness, and closeness centralities of each herb used in the mild stage treatment of COVID-19 were analyzed (Table 2). Sixteen herbs with a frequency of use of eight or more were identified, with Armeniacae semen amarum the most frequently used herb. In the modularity analysis, the 16 herbs were categorized into three modules (Figure 1). The first module (Figure 1, red nodes) included Poria sclerotium, Magnoliae cortex, Coicis semen, Citri reticulatae pericarpium, Armeniacae semen amarum, and Agastachis herba. The second module (Figure 1, green nodes) included Scutellariae radix, Platycodonis radix, Glycyrrhizae radix et rhizoma,
Forsythiae fructus, Bupleuri radix, and Arctii semen. The third module (Figure 1, blue nodes) included Ephedrae herba, Atractylodis rhizoma, Arecae semen, and Amomi tsao-ko fructus.

\section{Moderate Stage}

A total of 35 herbal formulae and 105 herbs were analyzed and 10 herb pairs with a high frequency of use (DMIM score $>0.85)$ were identified. The seven pairs of herbs with the highest DMIM scores were included PolyporusZingiberis rhizoma recens, Asari herba-Aurantii fructus, Alismatis rhizoma-Armeniacae semen amarum, Armeniacae semen amarum-Asteris radix, Belamcandae rhizoma-Cinnamomi ramulus, Zingiberis rhizoma recens-Asari herba, and Aurantii fructus-Citri reticulatae pericarpium (Table 1).

We also analyzed the frequency, degree, betweenness, and closeness centralities for each herb and identified 17 herbs with a frequency of use of seven or more for this disease stage. Once again, Armeniacae semen amarum was the herb with the highest frequency of use (Table 2). The 17 herbs were also categorized into three modules (Figure 2). The first module (Figure 2, red nodes) included Agastachis herba, Armeniacae semen amarum, Glycyrrhizae radix et rhizoma, Coicis semen, and Persicae semen. The second module (Figure 2, green nodes) included Gypsum fibrosum, Forsythiae fructus, Lonicerae flos, Mori radicis cortex, Scutellariae radix, and Lepidii seu descurainiae semen. The third module (Figure 2, blue nodes) included Cicadae periostracum, Ephedrae herba, Atractylodis rhizoma, Amomi tsao-ko fructus, Arecae semen, and Trichosanthis fructus.

\section{Severe Stage}

In the severe disease stage, we analyzed 42 herbal formulae comprising 127 herbs and identified 12 frequently used herb pairs (DMIM score > 0.88). The seven herb pairs with the highest DMIM score were Ossa draconis and Concha ostreae-Magnetitum, Magnetitum-Fluoritum, Dioscoreae rhizoma-Lilii bulbus, Lycii fructus-Psoraleae fructus, Bambusae succus-Jujubae fructus, Zingiberis rhizoma recens-Dioscoreae rhizoma, and Psoraleae fructusCuscutae semen (Table 1).

Following the frequency, degree, betweenness, and closeness centrality analyses for each herb, we identified 20 herbs with a frequency of use of nine or more at the severe disease stage. Bubali cornu was found to be the most frequently used herbs in the herbal formulae 
Table I Top Distance-Based Mutual Information Model (DMIM)Extracted Herbal Pairs

\begin{tabular}{|c|c|c|}
\hline $\begin{array}{l}\text { Disease } \\
\text { Stage }\end{array}$ & Herb Pairs & $\begin{array}{l}\text { DMIM } \\
\text { Score }\end{array}$ \\
\hline Mild & $\begin{array}{l}\text { Atractylodis rhizoma-Amomi tsao-ko } \\
\text { fructus } \\
\text { Arecae semen-Amomi tsao-ko fructus } \\
\text { Pinelliae rhizoma-Amomi fructus } \\
\text { rotundus } \\
\text { Coicis semen-Amomi fructus rotundus } \\
\text { Massa medicata fermentata-Crataegi } \\
\text { fructus } \\
\text { Polyporus-Alismatis rhizoma } \\
\text { Arctii semen-Platycodonis radix } \\
\text { Atractylodis rhizoma-Arecae semen } \\
\text { Forsythiae fructus-Platycodonis radix } \\
\text { Lonicerae flos-Arctii semen } \\
\text { Pinelliae rhizoma-Coicis semen } \\
\text { Amomi fructus rotundus-Tetrapanacis } \\
\text { medulla }\end{array}$ & $\begin{array}{l}0.90784 \\
0.90784 \\
0.90784 \\
0.90784 \\
0.90784 \\
\\
0.90784 \\
0.86461 \\
0.86461 \\
0.69737 \\
0.69737 \\
0.69737 \\
0.69737\end{array}$ \\
\hline Moderate & $\begin{array}{l}\text { Polyporus-Zingiberis rhizoma recens } \\
\text { Asari herba-Aurantii fructus } \\
\text { Alismatis rhizoma-Armeniacae semen } \\
\text { amarum } \\
\text { Armeniacae semen amarum-Asteris } \\
\text { radix } \\
\text { Belamcandae rhizoma-Cinnamomi } \\
\text { ramulus } \\
\text { Zingiberis rhizoma recens-Asari herba } \\
\text { Aurantii fructus-Citri reticulatae } \\
\text { pericarpium } \\
\text { Talcum-Artemisiae scopariae herba } \\
\text { Curcumae longae radix-Acori tatarinowii } \\
\text { rhizoma } \\
\text { Glycyrrhizae radix et rhizoma-Talcum }\end{array}$ & $\begin{array}{l}0.92644 \\
0.92644 \\
0.92644 \\
0.92644 \\
0.92644 \\
0.92644 \\
0.92644 \\
0.85237 \\
0.85237 \\
0.85237\end{array}$ \\
\hline Severe & $\begin{array}{l}\text { Ossa draconis and Concha ostreae- } \\
\text { Magnetitum } \\
\text { Magnetitum-Fluoritum } \\
\text { Dioscoreae rhizoma-Lilii bulbus } \\
\text { Lycii fructus-Psoraleae fructus } \\
\text { Bambusae succus-Jujubae fructus } \\
\text { Zingiberis rhizoma recens-Dioscoreae } \\
\text { rhizoma } \\
\text { Psoraleae fructus-Cuscutae semen } \\
\text { Uncariae ramulus cum uncis-Forsythiae } \\
\text { fructus } \\
\text { Forsythiae fructus-Phellodendri cortex } \\
\text { Lilii bulbus-Zingiberis rhizoma } \\
\text { Zingiberis rhizoma-Lycii fructus } \\
\text { Trogopterorum faeces-Allii macrostemi } \\
\text { bulbus }\end{array}$ & $\begin{array}{l}1.08217 \\
1.08217 \\
1.08217 \\
1.08217 \\
1.08217 \\
1.08217 \\
\\
1.08217 \\
0.92757 \\
\\
0.92757 \\
0.88148 \\
0.88148 \\
0.88148\end{array}$ \\
\hline
\end{tabular}

(Continued)
Table I (Continued).

\begin{tabular}{|l|l|l|}
\hline $\begin{array}{l}\text { Disease } \\
\text { Stage }\end{array}$ & Herb Pairs & $\begin{array}{l}\text { DMIM } \\
\text { Score }\end{array}$ \\
\hline Recovery & Ossa draconis and Concha ostreae- & 1.08217 \\
& Magnetitum & \\
& Magnetitum-Fluoritum & 1.08217 \\
& Dioscoreae rhizoma-Lilii bulbus & 1.08217 \\
& Lycii fructus-Psoraleae fructus & 1.08217 \\
& Bambusae succus-Jujubae fructus & 1.08217 \\
& Zingiberis rhizoma recens-Dioscoreae & 1.08217 \\
& rhizoma & \\
& Psoraleae fructus-Cuscutae semen & 1.08217 \\
& Uncariae ramulus cum uncis-Forsythiae & 0.92757 \\
& fructus & \\
& Forsythiae fructus-Phellodendri cortex & 0.92757 \\
& Lilii bulbus-Zingiberis rhizoma & 0.88148 \\
& Zingiberis rhizoma-Lycii fructus & 0.88148 \\
& Faeces trogopterori-Allii macrostemi & 0.88148 \\
& bulbus & \\
\hline
\end{tabular}

recommended for treating severe COVID-19 (Table 2). Similar to the other disease stages, the 20 herbs were categorized into three modules (Figure 3). The first module (Figure 3, red nodes) included Ginseng radix, Aconiti lateralis radix praeparata, Corni fructus, Bubali cornu, Moschus, Aquilariae lignum, Aucklandiae radix, Cinnabaris, and Bezoar bovis. The second module (Figure 3, green nodes) included Borneolum, Realgar, Coptidis rhizoma, Scutellariae radix, and Gardeniae fructus. The third module (Figure 3, blue nodes) included Gypsum fibrosum, Glycyrrhizae radix et rhizoma, Rhei radix et rhizoma, Armeniacae semen amarum, Trichosanthis fructus, and Ephedrae herba.

\section{Recovery Stage}

We analyzed a total of 29 herbal formulae and 94 herbs for the disease recovery stage. The twelve herb pairs with the highest frequency of use were identified (DMIM score > 1.08). The seven pairs of herbs with the highest DMIM score were Ossa draconis and Concha ostreaeMagnetitum, Magnetitum-Fluoritum, Dioscoreae rhizoma-Lilii bulbus, Lycii fructus-Psoraleae fructus, Bambusae succus-Jujubae fructus, Zingiberis rhizoma recens-Dioscoreae rhizoma, and Psoraleae fructusCuscutae semen. 
Table 2 Frequency, Degree, Closeness and Betweenness Centralities of Herbs

\begin{tabular}{|c|c|c|c|c|c|}
\hline \multirow[t]{2}{*}{ Rank/Stage } & \multirow[t]{2}{*}{ Herbal Medicine } & \multirow[t]{2}{*}{ Frequency, N (\%) } & \multicolumn{3}{|c|}{ Centrality } \\
\hline & & & Degree & Closeness & Betweenness \\
\hline \multicolumn{6}{|l|}{ Mild } \\
\hline I & Armeniacae semen amarum & $25(5.7)$ & 0.8 & 0.83333 & 0.39127 \\
\hline 2 & Magnoliae cortex & $20(4.6)$ & 0.53333 & 0.65217 & 0.10635 \\
\hline 3 & Glycyrrhizae radix et rhizoma & $19(4.4)$ & 0.33333 & 0.57692 & 0.00952 \\
\hline 4 & Forsythiae fructus & $19(4.4)$ & 0.53333 & 0.65217 & 0.17143 \\
\hline 5 & Platycodonis radix & $15(3.4)$ & 0.2 & 0.51724 & 0 \\
\hline 6 & Agastachis herba & $15(3.4)$ & 0.26667 & 0.5357 I & 0.13333 \\
\hline 7 & Ephedrae herba & $15(3.4)$ & 0.53333 & 0.65217 & 0.06111 \\
\hline 8 & Atractylodis rhizoma & $14(3.2)$ & 0.4 & 0.57692 & 0.01349 \\
\hline 9 & Scutellariae radix & $14(3.2)$ & 0.33333 & 0.57692 & 0.13333 \\
\hline 10 & Amomi tsao-ko fructus & $13(3.0)$ & 0.33333 & $0.5357 \mid$ & 0.00238 \\
\hline II & Coicis semen & $13(3.0)$ & 0.2 & 0.5 & 0 \\
\hline 12 & Citri reticulatae pericarpium & II (2.5) & 0.06667 & 0.35714 & 0 \\
\hline 13 & Arecae semen & II (2.5) & 0.26667 & 0.51724 & 0 \\
\hline 14 & Bupleuri radix & II (2.5) & 0.06667 & 0.375 & 0 \\
\hline 15 & Poria sclerotium & II (2.5) & 0.26667 & $0.5357 \mid$ & 0.00635 \\
\hline 16 & Arctii semen & $8(1.8)$ & 0.06667 & $0.4054 \mid$ & 0 \\
\hline \multicolumn{6}{|l|}{ Moderate } \\
\hline I & Armeniacae semen amarum & $31(6.9)$ & 0.875 & 0.88889 & 0.25139 \\
\hline 2 & Ephedrae herba & $30(6.7)$ & 0.9375 & 0.94118 & 0.37639 \\
\hline 3 & Gypsum fibrosum & $25(5.6)$ & 0.4375 & 0.64 & 0.01667 \\
\hline 4 & Glycyrrhizae radix et rhizoma & $21(4.7)$ & 0.5 & 0.66667 & 0.03681 \\
\hline 5 & Scutellariae radix & $21(4.7)$ & 0.4375 & 0.64 & 0.01875 \\
\hline 6 & Atractylodis rhizoma & $15(3.4)$ & 0.4375 & 0.61538 & 0 \\
\hline 7 & Lepidii seu descurainiae semen & $14(3.1)$ & 0.25 & 0.55172 & 0 \\
\hline 8 & Coicis semen & $13(2.9)$ & 0.1875 & 0.53333 & 0 \\
\hline 9 & Forsythiae fructus & $12(2.7)$ & 0.3125 & 0.59259 & 0 \\
\hline 10 & Agastachis herba & II (2.5) & 0.1875 & 0.53333 & 0 \\
\hline II & Amomi tsao-ko fructus & $10(2.2)$ & 0.25 & 0.55172 & 0 \\
\hline 12 & Arecae semen & $10(2.2)$ & 0.25 & 0.55172 & 0 \\
\hline 13 & Persicae semen & $9(2.0)$ & 0.125 & 0.51613 & 0 \\
\hline 14 & Cicadae periostracum & $8(1.8)$ & 0.125 & 0.51613 & 0 \\
\hline 15 & Lonicerae flos & $8(1.8)$ & 0.0625 & 0.4 & 0 \\
\hline 16 & Mori radicis cortex & $8(1.8)$ & 0.1875 & 0.55172 & 0 \\
\hline 17 & Trichosanthis fructus & $7(1.6)$ & 0.0625 & 0.5 & 0 \\
\hline \multicolumn{6}{|l|}{ Severe } \\
\hline I & Bubali cornu & $23(3.9)$ & 0.78947 & 0.82609 & 0.4347 \\
\hline 2 & Glycyrrhizae radix et rhizoma & $22(3.8)$ & 0.31579 & 0.59375 & 0.12573 \\
\hline 3 & Moschus & $21(3.6)$ & 0.68421 & 0.65517 & 0.07115 \\
\hline 4 & Gypsum fibrosum & $21(3.6)$ & 0.31579 & 0.59375 & 0.2271 \\
\hline 5 & Aconiti lateralis radix praeparata & $20(3.4)$ & 0.36842 & 0.57576 & 0.02437 \\
\hline 6 & Cinnabaris & $20(3.4)$ & 0.68421 & 0.65517 & 0.07115 \\
\hline 7 & Corni fructus & $17(2.9)$ & 0.31579 & 0.55882 & 0.01462 \\
\hline 8 & Ginseng radix & $14(2.4)$ & 0.26316 & 0.51351 & 0 \\
\hline 9 & Borneolum & $13(2.2)$ & 0.42105 & 0.55882 & 0.00585 \\
\hline 10 & Scutellariae radix & $13(2.2)$ & 0.36842 & 0.54286 & 0 \\
\hline II & Armeniacae semen amarum & $13(2.2)$ & 0.26316 & 0.43182 & 0.01949 \\
\hline
\end{tabular}

(Continued) 
Table 2 (Continued).

\begin{tabular}{|c|c|c|c|c|c|}
\hline \multirow[t]{2}{*}{ Rank/Stage } & \multirow[t]{2}{*}{ Herbal Medicine } & \multirow[t]{2}{*}{ Frequency, N (\%) } & \multicolumn{3}{|c|}{ Centrality } \\
\hline & & & Degree & Closeness & Betweenness \\
\hline 12 & Rhei radix et rhizoma & $12(2.0)$ & 0.15789 & 0.39583 & 0 \\
\hline 13 & Aucklandiae radix & II (I.9) & 0.21053 & 0.5 & 0 \\
\hline 14 & Gardeniae fructus & II (1.9) & 0.36842 & 0.54286 & 0 \\
\hline 15 & Ephedrae herba & II (1.9) & 0.15789 & 0.41304 & 0 \\
\hline 16 & Aquilariae lignum & $10(1.7)$ & 0.21053 & 0.5 & 0 \\
\hline 17 & Realgar & $10(1.7)$ & 0.42105 & 0.55882 & 0.00585 \\
\hline 18 & Coptidis rhizoma & $10(1.7)$ & 0.36842 & 0.54286 & 0 \\
\hline 19 & Bezoar bovis & $9(1.5)$ & 0.21053 & 0.5 & 0 \\
\hline 20 & Trichosanthis fructus & $9(1.5)$ & 0.15789 & 0.39583 & 0 \\
\hline \multicolumn{6}{|l|}{ Recovery } \\
\hline 1 & Glycyrrhizae radix et rhizoma & $20(6.6)$ & 0.69231 & $0.7647 \mid$ & 0.45 \\
\hline 2 & Poria sclerotium & $17(5.6)$ & 0.69231 & $0.7647 \mid$ & 0.19957 \\
\hline 3 & Ophiopogonis radix & $17(5.6)$ & 0.38462 & 0.61905 & 0.07564 \\
\hline 4 & Citri reticulatae pericarpium & $16(5.3)$ & 0.69231 & $0.7647 \mid$ & 0.19957 \\
\hline 5 & Atractylodis macrocephalae rhizoma & $12(4.0)$ & 0.23077 & 0.56522 & 0 \\
\hline 6 & Codonopsis radix & II (3.7) & 0.46154 & 0.65 & 0.06239 \\
\hline 7 & Lophatheri herba & $9(3.0)$ & 0.15385 & 0.46429 & 0 \\
\hline 8 & Amomi fructus & $9(3.0)$ & 0.30769 & 0.5 & 0 \\
\hline 9 & Pseudostellariae radix & $8(2.7)$ & 0.15385 & 0.46429 & 0 \\
\hline 10 & Astragali radix & $8(2.7)$ & 0.07692 & 0.44828 & 0 \\
\hline II & Pinelliae rhizoma & $7(2.3)$ & 0.07692 & 0.44828 & 0 \\
\hline 12 & Pinelliae rhizoma praeparatum & $7(2.3)$ & 0.23077 & $0.48 \mid 48$ & 0 \\
\hline 13 & Astragali radix praeparata cum melle & $7(2.3)$ & 0.30769 & 0.5 & 0 \\
\hline 14 & Agastachis herba & $6(2.0)$ & 0.15385 & 0.46429 & 0 \\
\hline
\end{tabular}

After analyzing the frequency, degree, betweenness, and closeness centralities for each herb, we identified 14 herbs with a frequency of use of six or more, and Glycyrrhizae radix et rhizoma had the highest frequency of use (Table 2). The 14 herbs were categorized into two modules based on our modularity analysis (Figure 4). The first module (Figure 4, red nodes) included Pinelliae rhizoma, Ophiopogonis radix, Lophatheri herba, Glycyrrhizae radix et rhizoma, Pseudostellariae radix, and Astragali radix. The second module (Figure 4, green nodes) included Poria sclerotium, Citri reticulatae pericarpium, Pinelliae rhizoma praeparatum, Codonopsis radix, Atractylodis macrocephalae rhizoma, Astragali radix praeparata cum melle, Amomi fructus, and Agastachis herba.

\section{Discussion}

The results of our network analyses provide insights into the prescription pattern, therapeutic logic, and combinations of herbs used in the herbal formulae recommended for the treatment of each stage of COVID-19.

To understand the combination rule embedded in the herbal formulae recommended for COVID-19, we used DMIM scores to compute the tendency of two herbs to form a pair. Interestingly, we found that the herb Glycyrrhizae radix et rhizoma only appears in one herb pair (Glycyrrhizae radix et rhizoma-Talcum, Table 1), even though this herb appears in the list of herbs with high frequency of use for every disease stage (Table 2). This showed that the DMIM method was able to avoid the pitfalls of other analytical methods by quantitatively computing the frequency and MI value of the herbs, and the "between-herb-distance" in the herbal formulae.

In the theory of traditional medicine (TM), the syndrome differentiation of the four stages of COVID-19 includes "cold-dampness and stagnation lung syndrome" and "dampness and heat-accumulation lung syndrome" for the mild stage; "dampness and stagnation lung 


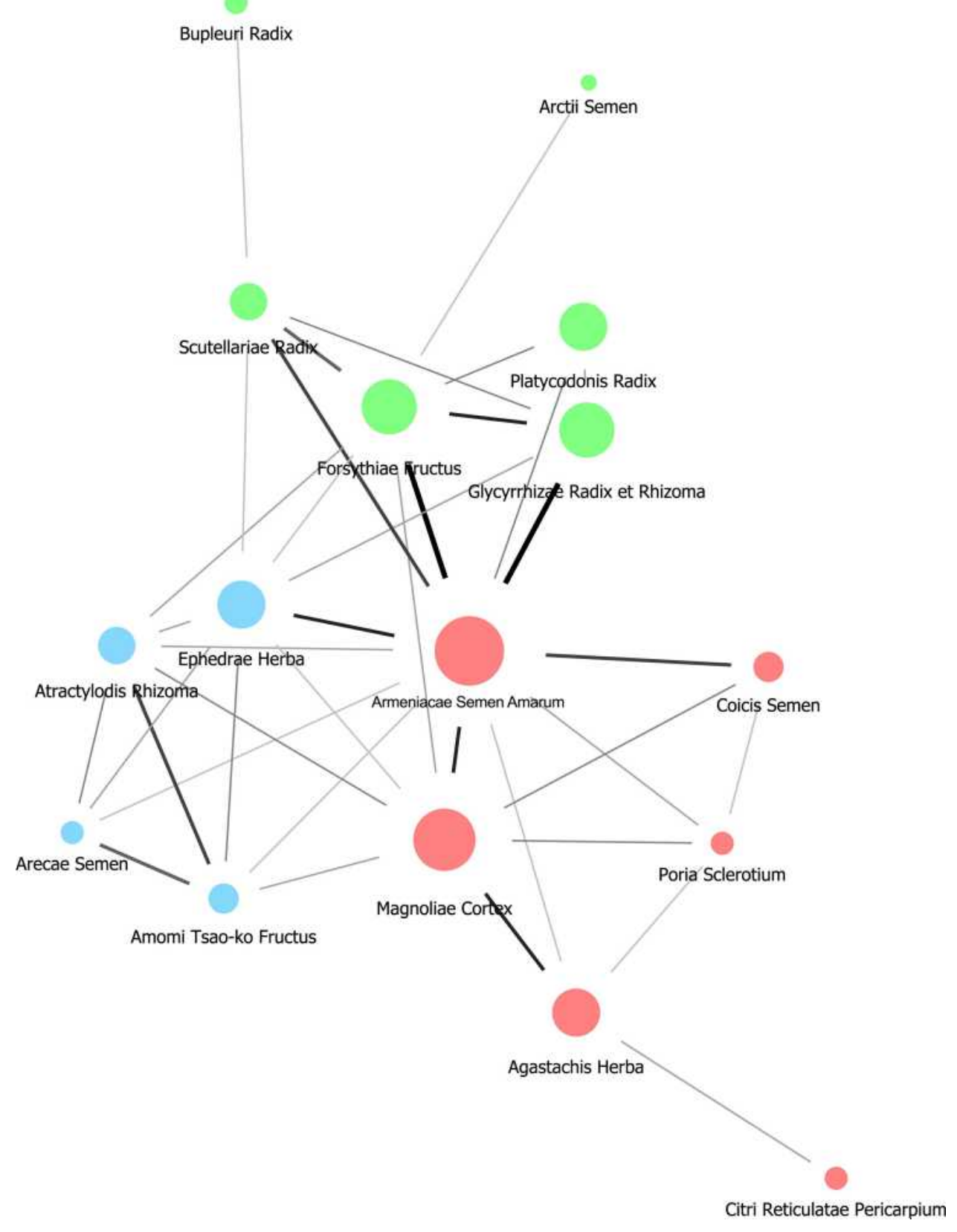

Figure I Herb network for mild stage.

syndrome" and "cold dampness lung syndrome" for the moderate stage; "plague poison and lung-closing syndrome", " syndrome of flaring heat in Qifen and Yingfen", and "syndrome of inner blocking causing collapse" for severe stage, and "lung and spleen Qi deficiency syndrome" and "Qi and Yin deficiency syndrome" for recovery stage. ${ }^{19}$ In terms of herbal medicine, herbs can be generally classified into cold/cool herbs and hot/ warm herbs. Cold-natured herbs have Yin properties such as clearing heat, purging fire, and dispelling toxic and mainly used for heat-related syndromes, whereas warmnatured herbs have Yang properties such as expelling cold, tonifying Qi, and restoring Yang and mainly used for cold-related syndromes. ${ }^{20}$ Therefore, the therapeutic principle and herbal formulae/herbs used vary across each disease stage according to the syndrome differentiation.

In the mild stage of COVID-19, the herbs with the highest frequency of use were mainly warm-natured and can be classified into lung, spleen, and stomach meridians. 


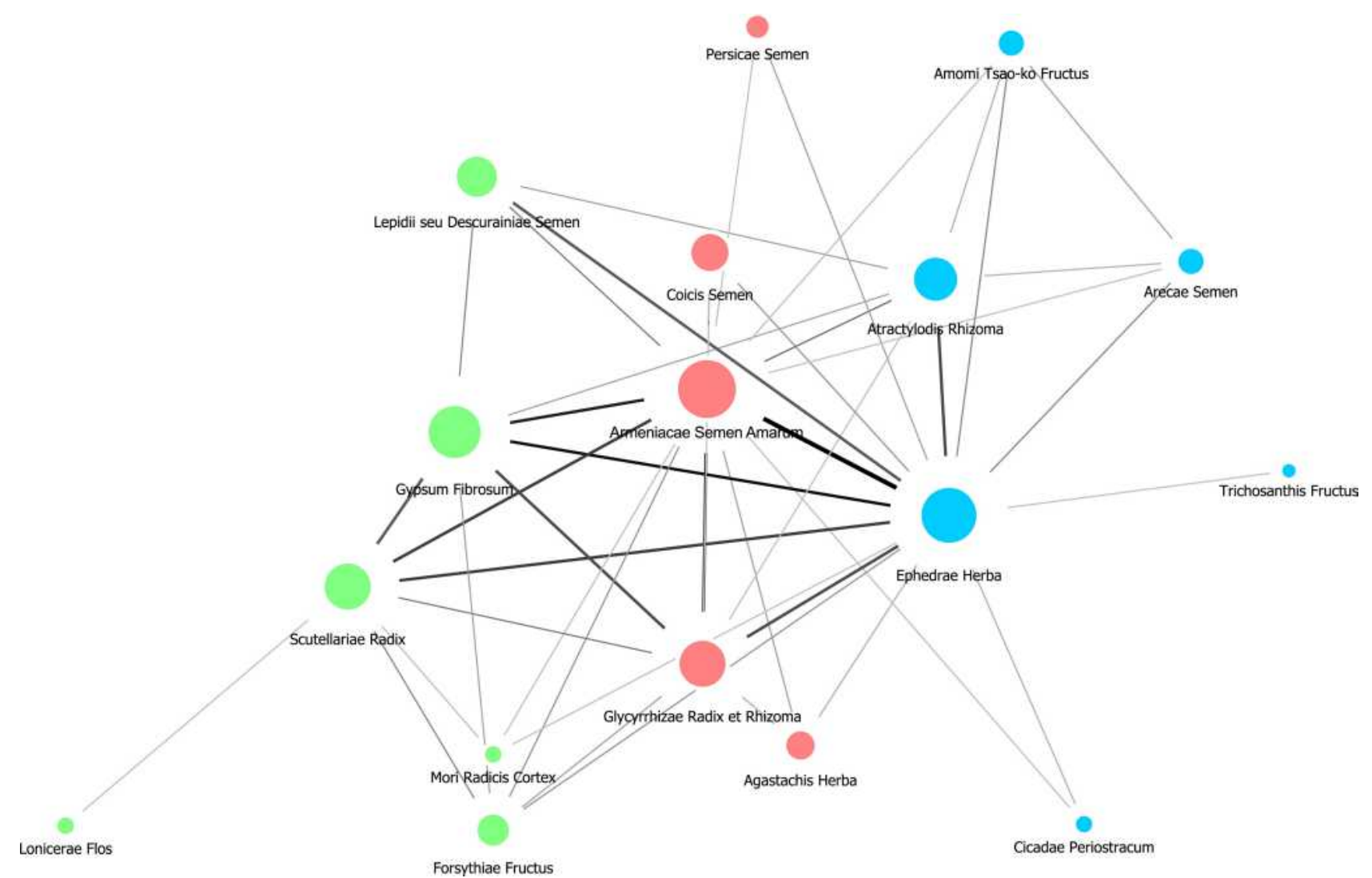

Figure 2 Herb network for moderate stage.

The main TM therapeutic principle of these herbs can be summarized as releasing the exterior and resolving dampness, corresponding with the symptoms of mild COVID19 such as fever (ie due to external pathogenic factors) and cough (ie due to dampness). In contrast, the herbs with high frequency of use at the moderate stage of COVID-19 are partly warm-natured and partly cold-natured. Similar to the mild stage, these herbs can be mainly classified into lung, spleen, and stomach meridians. The main TM therapeutic principle for the moderate stage can be summarized as clearing heat, drying dampness, and suppressing the cough. This also corresponds with the symptoms of moderate COVID-19 resulting from pathogenic factors of heat, such as fever, sore throat, and pneumonia, and dampness, such as cough, shortness of breath, myalgia, fatigue, and diarrhea.

For both mild and moderate stages, the herb Armeniacae semen amarum was the most frequently recommended. Based on our findings, we inferred that most herbal formulae recommended in the mild and moderate stages are modified using "Ma Xing Shi Gan" decoction as the basic formula. "Ma Xing Shi Gan" decoction has been reported to have a broad spectrum of inhibitory activity against the entry of the influenza virus and have a significant effect of reducing pulmonary inflammation. ${ }^{21,22 ~ " M a ~ X i n g ~ S h i ~ G a n " ~}$ decoction is composed of four herbs including Ephedrae herba, Armeniacae semen amarum, Gypsum fibrosum, and Glycyrrhizae radix et rhizoma. A study exploring the active compounds of Ephedrae herba found several key components such as $\beta$-sitosterol, estrone, and stigmasterol have potential therapeutic effects on viral-induced respiratory infections. ${ }^{23}$ Wang et al also revealed that Armeniacae semen amarum has nine active compounds regulating eight targets, with amygdalin as the major effective component and a candidate compound for COVID-19 treatment. ${ }^{24}$ The herb Gypsum fibrosum is also shown to have an antipyretic effect with calcium as its main active component. ${ }^{25}$ A study by Niu et al also identified that compounds such as quercetin, glabridin, and gallic acid present in Glycyrrhizae radix et rhizoma may regulate angiotensin-converting enzyme 2 (ACE2) for the treatment of COVID-19. ${ }^{26}$

For the severe stage of COVID-19, the herbs with high frequency of prescription were partly warm-natured and partly cold-natured herbs, including herbs with an 


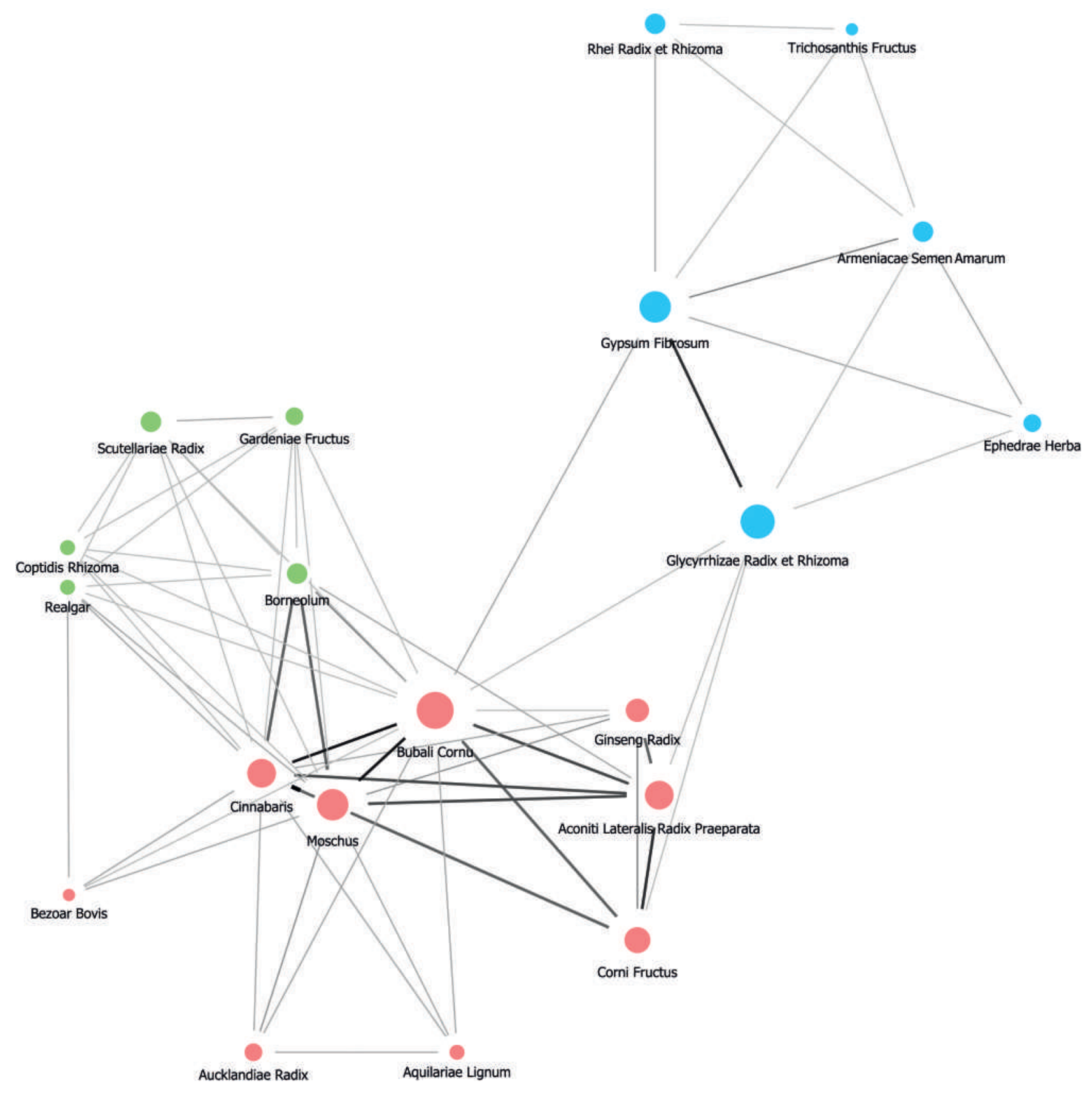

Figure 3 Herb network for severe stage.

extreme-warm-nature and extreme-cold-nature. These herbs can be primarily classified into lung, spleen, and heart meridians. The main TM therapeutic principle can be characterized as clearing heat, dispelling phlegm, and opening the orifices, corresponding to the symptoms of severe COVID-19 such as pneumonia (ie due to heat), comatose due to respiratory failure and shock (ie due to excessive phlegm and closing of orifices). According to a retrospective cohort study, the most frequently observed complication in COVID-19 patients is sepsis, followed by multiple organ failure, and septic shock. ${ }^{27}$ This elucidates our findings as the high frequency of recommendation and pairing of herbs in the severe stage are mainly compositions of herbal medicines like "Angong Niuhuang" pill, "Zhi Bao Dan", “Zi Xue San”, and "Su He Xiang” pill, which are frequently prescribed during life-threatening conditions. Among the top herbs used in the severe stage, Bubali cornu has also shown to have antipyretic 


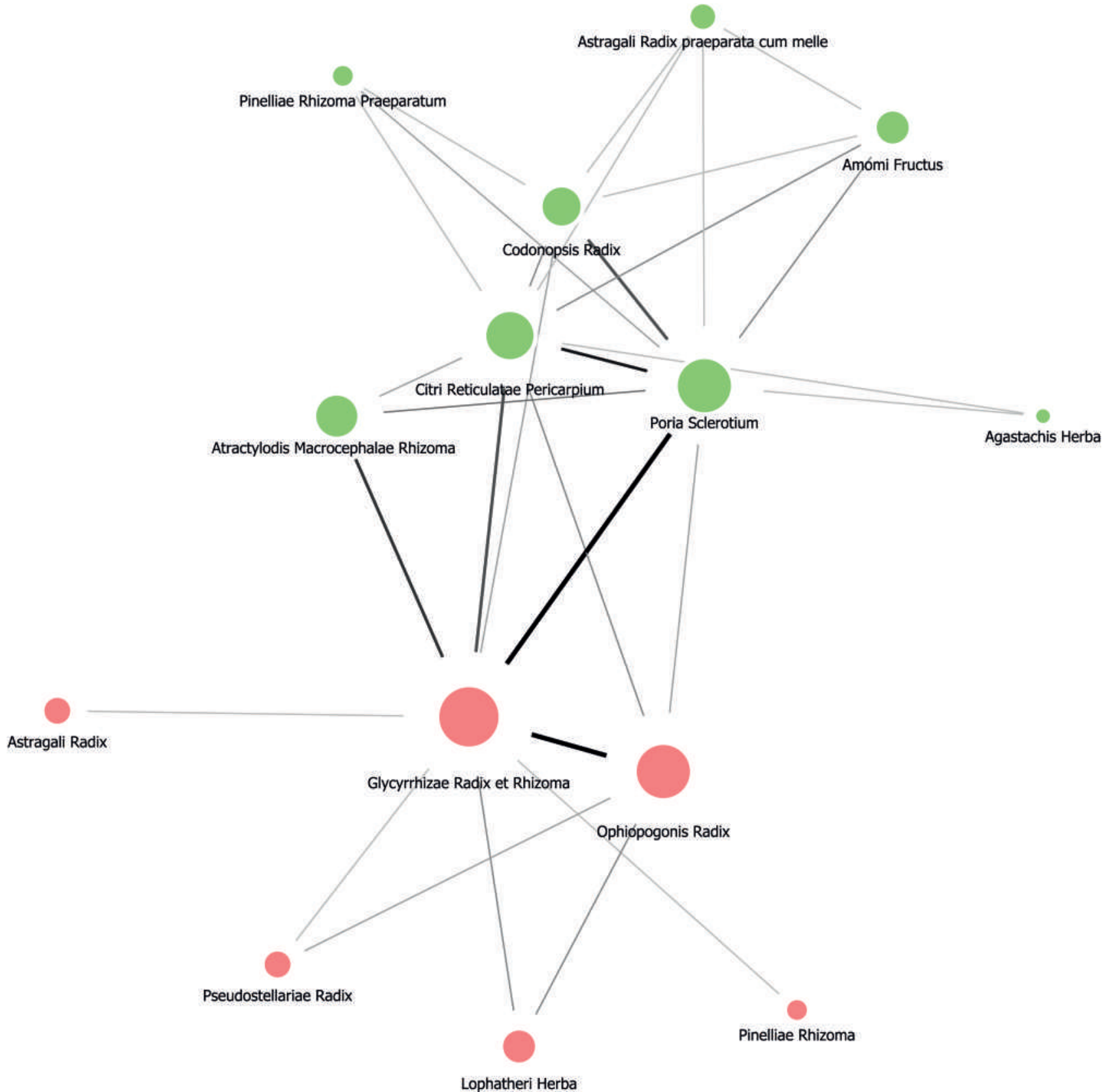

Figure 4 Herb network for recovery stage.

compound $^{28}$ and Aconiti lateralis radix praeparata has played a potential role in treating heart failure due to its high alkaloid contents. ${ }^{29}$

For the recovery stage, the herbs with high frequency of prescription were mainly warm-natured and can be classified as lung and spleen meridians. The main TM therapeutic principle can be summarized as tonifying qi and resolving dampness. In this stage, the symptoms are often milder after treatment, where the fever has subsided and respiratory symptoms, such as a cough or shortness of breath, have improved. Hence, the recommended herbs and TM therapeutic principles during the recovery process are focused on nourishing the body and improving prolonged mild symptoms. A study by Ko et al reveals that tonifying herbs such as Glycyrrhizae radix et rhizoma, Ophiopogonis radix, Atractylodis macrocephalae rhizoma, Codonopsis radix, and Pseudostellariae radix could enhance ATP-generation capacity. ${ }^{30}$ This also corresponds with our findings as these herbs are also among the top frequently used herbs in the recovery stage. 
In addition, we found that the herbs with the highest frequency of use for every stage in our analysis comprised of the composition of herbs in the "Qingfei Paidu" decoction. The "Qingfei Paidu" decoction is a basic herbal formula consisting of 21 herbs included in the Chinese treatment guidelines as the primarily recommended formula that applies to all stages of COVID-19. The "Qingfei Paidu" decoction is reported to have 129 active compounds that can act on many targets to inhibit the entry and replication of SARSCoV-2, suggesting a potential effect in treating COVID-19. ${ }^{22,31,32}$ Recent studies have shown that the "Qingfei Paidu" decoction has significant effects on alleviating the symptoms and reducing multi-organ failure in COVID-19. ${ }^{33-35}$ Notably, "Ma Xing Shi Gan" decoction is part of the fundamental composition in the "Qingfei Paidu" decoction and may contribute to the overall efficacy of the "Qingfei Paidu" decoction. ${ }^{22}$ Hence, we believe that the "Qingfei Paidu" decoction comprised of the herbs with high frequency of use across all disease stages and the modification of "Ma Xing Shi Gan" decoction, which is also the core herbal composition of "Qingfei Paidu" decoction, is the main herbal formula recommended for treatment of COVID-19.

\section{Conclusion}

Our results present the preliminary prescription pattern, therapeutic logic, and herbal formulae recommended in the treatment guidelines for COVID-19 using a network analysis approach. As there are numerous herb pairs with similar DMIM scores across the disease stage, we are unable to identify the herb pair with the highest potential in treating COVID-19. However, we found that the four herbs of "Ma Xing Shi Gan" decoction including Ephedrae herba, Armeniacae semen amarum, Gypsum fibrosum, and Glycyrrhizae radix et rhizoma seem to each play a potential role in every stage of COVID-19. Future studies may consider focusing on the mechanism and metabolic pathway for these herbs for the treatment of COVID-19. We hope that this study may provide insights and research ideas for related clinical research in the future.

\section{Data Sharing Statement}

Data will be made available upon reasonable request.

\section{Author Contributions}

All authors (L.A., H.W.L., A.K., J.Y.C., and M.S.L) made a significant contribution to the work reported, whether that is in the conception, study design, execution, acquisition of data, analysis and interpretation, or in all these areas; took part in drafting, revising or critically reviewing the article; gave final approval of the version to be published; have agreed on the journal to which the article has been submitted; and agree to be accountable for all aspects of the work.

\section{Funding}

This study was supported by the Korea Institute of Oriental Medicine (KSN2021210).

\section{Disclosure}

The authors report no conflicts of interest in this work.

\section{References}

1. Lai KKR, Wu J, Harris R, et al. Coronavirus map: tracking the spread of the outbreak. The New York Times Web site; Updated March 30, 2021. Available from: https://www.nytimes.com/interactive/2020/ world/coronavirus - maps.html action $=$ click $\& \operatorname{module}=$ RelatedLinks\&pgtype=Article. Accessed March 30, 2021.

2. Yang Y, Islam MS, Wang J, Li Y, Chen X. Traditional Chinese medicine in the treatment of patients infected with 2019-new coronavirus (SARS-CoV-2): a review and perspective. Int $J$ Biol Sci. 2020;16(10):1708-1717. doi:10.7150/ijbs.45538

3. Remali J, Aizat WMA. Review on plant bioactive compounds and their modes of action against coronavirus infection. Front Pharmacol. 2021;11. doi:10.3389/fphar.2020.589044

4. Liu M, Gao Y, Yuan Y, et al. Efficacy and safety of integrated traditional Chinese and western medicine for corona virus disease 2019 (COVID-19): a systematic review and meta-analysis. Pharmacol Res. 2020;158:104896. doi:10.1016/j.phrs.2020.104896

5. Ang L, Song E, Lee HW, Lee MS. Herbal medicine for the treatment of coronavirus disease 2019 (COVID-19): a systematic review and meta-analysis of randomized controlled trials. J Clin Med. 2020;9 (5): 1583. doi: $10.3390 / \mathrm{jcm} 9051583$

6. Wu YQ, Zou L, Yu X, et al. Clinical effects of integrated traditional Chinese and western medicine on COVID-19: a systematic review. Shanghai J Tradit Chin Med. 2020;1-8.

7. Börner K, Sanyal S, Vespignani A. Network science. Annu Rev Inform Sci. 2007;41:537-607.

8. Hawe P, Webster C, Shiell A. A glossary of terms for navigating the field of social network analysis. J Epidemiol Community Health. 2004;58(12):971-975. doi:10.1136/jech.2003.014530

9. Lee SH, Kim CE, Lee IS, et al. Network analysis of acupuncture points used in the treatment of low back pain. Evid Based Complement Alternat Med. 2013;2013:402180.

10. Park JY, Lee SH, Kim SY, Park HJ. Literature review and network analysis on the pain disease approach of Saam acupuncture method. Korean J Acupunct. 2017;34(2):88-99. doi:10.14406/acu.2017.011

11. Yang DH, Kang JH, Park YB, Park YJ, Oh HS, Kim SB. Association rule mining and network analysis in oriental medicine. PLoS One. 2013;8(3):e59241. doi:10.1371/journal.pone.0059241 
12. Li Y, Li R, Ouyang Z, Li S. Herb network analysis for a famous TCM doctor's prescriptions on treatment of rheumatoid arthritis. Evid Based Complement Alternat Med. 2015;2015:451319.

13. Liang H, Ruan H, Ouyang Q, Lai L. Herb-target interaction network analysis helps to disclose molecular mechanism of traditional Chinese medicine. Sci Rep. 2016;6(1):36767. doi:10.1038/srep36767

14. Lin YJ, Liang WM, Chen CJ, et al. Network analysis and mechanisms of action of Chinese herb-related natural compounds in lung cancer cells. Phytomedicine. 2019;58:152893. doi:10.1016/j. phymed.2019.152893

15. Ang L, Lee HW, Choi JY, Zhang J, Lee MS. Herbal medicine and pattern identification for treating COVID-19: a rapid review of guidelines. Integr Med Res. 2020;9(2):100407.

16. Li S, Zhang B, Jiang D, Wei Y, Zhang N. Herb network construction and co-module analysis for uncovering the combination rule of traditional Chinese herbal formulae. BMC Bioinform. 2010;11(Suppl 11): S6. doi:10.1186/1471-2105-11-S11-S6

17. Newman MEJ. Finding community structure in networks using the eigenvectors of matrices. Phys Rev E. 2006;74(3):036104. doi:10.1103/PhysRevE.74.036104

18. Freeman LC. Centrality in social networks conceptual clarification. Soc Networks. 1978;1(3):215-239.

19. An X, Zhang Y, Duan L, et al. The direct evidence and mechanism of traditional Chinese medicine treatment of COVID-19. Biomed Pharmacother. 2021;137:111267. doi:10.1016/j.biopha.2021.111267

20. Ergil MC, Ergil KV, Mayor DF. Pocket Atlas of Chinese Medicine. 1st ed. New York: Thieme; 2009.

21. Hsieh CF, Lo CW, Liu CH, et al. Mechanism by which ma-xing-shigan-tang inhibits the entry of influenza virus. $J$ Ethnopharmacol. 2012;143(1):57-67. doi:10.1016/j.jep.2012.05.061

22. Yang R, Liu H, Bai C, et al. Chemical composition and pharmacological mechanism of Qingfei Paidu Decoction and Ma Xing Shi Gan Decoction against Coronavirus Disease 2019 (COVID-19): in silico and experimental study. Pharmacol Res. 2020;157:104820. doi:10.1016/j.phrs.2020.104820

23. Gao K, Song YP, Song A. Exploring active ingredients and function mechanisms of Ephedra-bitter almond for prevention and treatment of Corona virus disease 2019 (COVID-19) based on network pharmacology. BioData Min. 2020;13(1):19. doi:10.1186/s13040020-00229-4

24. Wang Y, Gu W, Kui F, et al. The mechanism and active compounds of semen armeniacae amarum treating coronavirus disease 2019 based on network pharmacology and molecular docking. Food Nutr Res. 2021;65. doi:10.29219/fnr.v65.5623
25. Luo CH, Ma LL, Liu HM, et al. Research progress on main symptoms of novel coronavirus pneumonia improved by traditional Chinese medicine. Front Pharmacol. 2020;11:1448. doi:10.3389/ fphar.2020.556885

26. Niu W, Wu F, Cui H, et al. Network pharmacology analysis to identify phytochemicals in traditional Chinese medicines that may regulate ACE2 for the treatment of COVID-19. Evid Based Complement Alternat Med. 2020;2020:7493281. doi:10.1155/2020/ 7493281

27. Zhou F, Yu T, Du R, et al. Clinical course and risk factors for mortality of adult inpatients with COVID-19 in Wuhan, China: a retrospective cohort study. Lancet. 2020;395(10229):1054-1062. doi:10.1016/S0140-6736(20)30566-3

28. Ma LL, Liu HM, Luo CH, et al. Fever and antipyretic supported by traditional Chinese medicine: a multi-pathway regulation. Front Pharmacol. 2021;12(253). doi:10.3389/fphar.2021.583279

29. He YN, Zhang DK, Lin JZ, et al. Cardiac function evaluation for a novel one-step detoxification product of Aconiti Lateralis Radix Praeparata. Chin Med. 2018;13(1):62.

30. Ko KM, Leon TYY, Mak DHF, Chiu PY, Du Y, Poon MKT. A characteristic pharmacological action of 'Yang-invigorating' Chinese tonifying herbs: enhancement of myocardial ATPgeneration capacity. Phytomedicine. 2006;13(9):636-642. doi:10.1016/j.phymed.2006.02.007

31. Li C, Wang L, Ren L. Antiviral mechanisms of candidate chemical medicines and traditional Chinese medicines for SARS-CoV-2 infection. Virus Res. 2020;286:198073. doi:10.1016/j. virusres.2020.198073

32. Ren W, Ma Y, Wang R, et al. Research advance on Qingfei Paidu Decoction in prescription principle, mechanism analysis and clinical application. Front Pharmacol. 2021;11. doi:10.3389/ fphar.2020.589714

33. Xin S, Cheng X, Zhu B, et al. Clinical retrospective study on the efficacy of Qingfei Paidu decoction combined with Western medicine for COVID-19 treatment. Biomed Pharmacother. 2020;129:110500. doi:10.1016/j.biopha.2020.110500

34. Liu N, Li S, Fan K, et al. The prevention and treatment of COVID-19 with Qingfei Paidu decoction in shanxi China. TMR Mod Herb Med. 2020;3:173-177.

35. Zhai J, Yin J. Review: the vital role of traditional Chinese medicine (TCM) in the prevention and treatment of COVID-19 and the etiology and pathogenic mechanism of COVID-19 based on TCM theory. Asian J Tradit Med. 2020;15(3):127-135.
Infection and Drug Resistance

\section{Publish your work in this journal}

Infection and Drug Resistance is an international, peer-reviewed openaccess journal that focuses on the optimal treatment of infection (bacterial, fungal and viral) and the development and institution of preventive strategies to minimize the development and spread of resistance. The journal is specifically concerned with the epidemiology of antibiotic resistance and the mechanisms of resistance development and diffusion in both hospitals and the community. The manuscript management system is completely online and includes a very quick and fair peerreview system, which is all easy to use. Visit http://www.dovepress.com/ testimonials.php to read real quotes from published authors. 